\title{
Reduction of territorial fire risks based on methods of active systems theory
}

\author{
Vladimir Minaev ${ }^{1}$, Alexander Faddeev ${ }^{2}$, Tuan $\mathrm{Dao}^{3}$ and Phan Tuan $\mathrm{Anh}^{4 *}$ \\ ${ }^{1}$ Bauman Moscow State Technical University, 105005, Baumanskaya 2-ya str., 5, Moscow, Russia \\ ${ }^{2}$ The Academy of Law and Management of the Federal Penal Service of Russia, 390036, Sennaya str., \\ 1, Ryazan, Russia \\ ${ }^{3}$ The University of Fire Fighting and Prevention, 10000, 243 Khuat Duy Tien, Hanoi, Vietnam \\ ${ }^{4}$ Moscow State University of Civil Engineering, Yaroslavskoe shosse, 26, Moscow, 129337, Russia
}

\begin{abstract}
Subject of the study: Models of relationships between specific index for the number of deaths and injuries in fires and specific load by fires are studied. A complex specific indicator (the number of deaths and injuries in fires) is formed. Thereupon, based on the principles of active systems theory, a target function for optimal territorial distribution of human resources of fire service in clusters - groups of homogeneous provinces in terms of fire risks - is constructed.

Goal of the study: The article is aimed at justifying and constructing complex criteria for optimal management of the human resources of the fire service.

Materials and methods: Methodological basis for fire risks assessment is the integral fire risks theory, and human resources management is the theory of active systems. The dynamics of fire risk indicators in Vietnam from 2006 to 2016 is considered.

Results: The optimal distribution of human resources allows a $10-12 \%$ reduction in the complex specific indicator of risks.

Conclusions: The obtained solutions make it possible to develop practical recommendations for authorities, industries, including construction industry, and fire service, on improving management of territorial human resources taking into account fire risks, enhancing legal and regulatory support for insurance in construction industry.
\end{abstract}

\section{Introduction}

The tasks of reducing fire risks is relevant for each branch of the economy. Construction industry is no exception, therefore, the improvement of the management of the resources of the territorial fire service is great importance for it [1-3], being one of the crucial aspects in the general range of its effective interaction with divisions of related industries and services.

A sufficiently fruitful scientific concept, which describes the activity of fire service system as "processing" of variables input vector into a specified output vector of results in accordance with a specific operational and service "technology" using necessary quantity

\footnotetext{
${ }^{*}$ Corresponding author: ptuananh26789@ gmail.com
} 
and quality of human resources is proposed and justified in the paper [4]. For example, an input variable can be the number of fires in construction, a result - an output - the economic damage for construction industry resulted from fires; an input - the number of violations of fire safety in construction, an output - the number of orders and penalties imposed on construction companies.

As shown in the mentioned paper [4], the effective solution of the tasks of managing the resources of the fire service is associated with a quantitative study of the dependencies between the specific indicators, which are related to the ensuring fire safety in the aspect of time and territory.

\section{Results of the study}

\subsection{Modeling of interrelationships of activities' indicators of fire service}

Relationship of specific index (attributable per one firefighter) for the number of deaths in fires (SIDF) with specific load by fires (SLF) is considered. This dependence is well described by the following exponential curve (explanability, i.e., squared correlation coefficient is equal to $73 \%$ ):

$$
e=0,33 \cdot \exp (107,23 \cdot h) \text {. }
$$

High explanability (76\%) characterizes empirical relationship of the specific index for the number of injuries in fires (SIIF) with specific load by fires (SLF), which is described by the exponential curve.

$$
e=0,31 \cdot \exp (90,3 \cdot h) \text {. }
$$

Comparing the relationship between the specific activities' indicators of fire service SIDF, SIIF and SLF shows that they are ascending.

Based on the behavior of the specific indicators, let's form a complex specific indicator that takes into account both deaths and injuries in fires.

To do this, let's add up the specific indicators SIDF and SIIF, which are weighted by standardized coefficients:

$$
s_{k}=\alpha \cdot d_{k}+\beta \cdot w_{k},
$$

where $k=1,2, \ldots, K-$ the number of observation points (years), $K-$ the total number of years of observation equal to 10 years; $\alpha$ - weight of the specific indicator $d_{k} ; \beta$ - specific indicator weight $w_{k}$. The amount of weighting coefficients is constrained:

$$
\alpha+\beta=1
$$
follows:

The problem of finding weighting coefficients $\alpha, \beta$ and model parameters was set as

$$
\mathrm{S}=\max _{\alpha, \beta, \vec{p}} \mathrm{R}^{2}[s(\widehat{\vec{p}, \alpha, \beta})]
$$

where $\mathrm{S}-$ the value of the functional calculated as a maximum of the explanability coefficient by varying model parameters $(\vec{p}-$ the vector $)$ chosen from the class of exponential curves and weighting coefficients $\alpha$ and $\beta$ with constraint (4).

Real data calculations have shown that the best model for Vietnam is formed as follows:

$$
s=(0,555 \cdot d+0,445 \cdot w)=0,318 \cdot \exp (101,04 \cdot h) .
$$

Maximum value of the explanability coefficient is equal to $82 \%$ for $\alpha=0.555$ and $\beta=$ 0.445 .

Figure 1 shows empirical data and theoretical description of the relationship between the complex specific indicator "s" and SLF in Vietnam in 2006 - 2016. 


\subsection{The task of optimal ensuring of human resources for the fire services of territorial clusters}

Figure 2 schematically shows a process of operational and service activities of fire service's personnel subsystems in terms of "input - human resources - output", which should be reflected in explicit mathematical description of "operational and service technology" in each case.

It should be noted that the territorial clusters, which are homogeneous in term of fire risks, are usually found by using various methods of cluster analysis. With regard to Vietnam, such a problem is successfully solved [5], and results of the solution are described in this collection.

Based on the results of the paper [4], let's introduce the target of function $\varphi=\varphi(\bar{X}, \bar{Y}, \vec{\gamma})$, which reflects effectiveness of the use fire service's resources. With regard to the $k$-th cluster in Vietnam, let's write it as follows:

$$
\varphi_{k}=\varphi_{k}\left(F_{k}, R_{k}, \bar{\gamma}_{k}\right)
$$

where the $F_{k}$ - the number of fires in the $k$-th territorial cluster, $R_{k}$ - the number of firefighter in the $k$-th cluster, $\bar{\gamma}_{k}-$ the parameters' vector of target function in the $k$-th cluster.

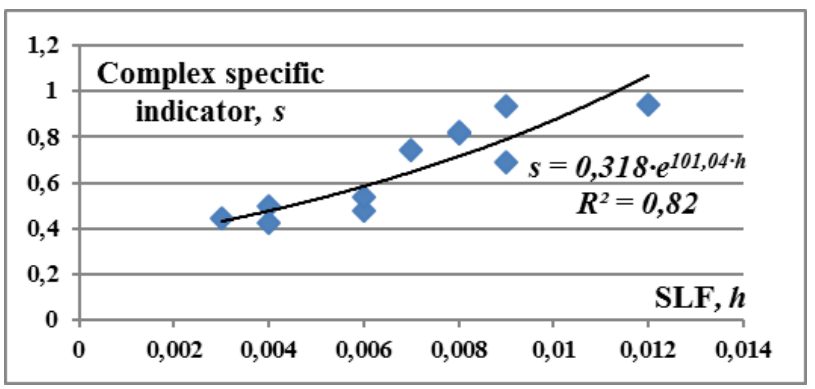

Fig. 1. Empirical data (diamonds) and theoretical description (solid curve) of the relationship between the complex specific indicator s and SLF in Vietnam in $2006-2016$.

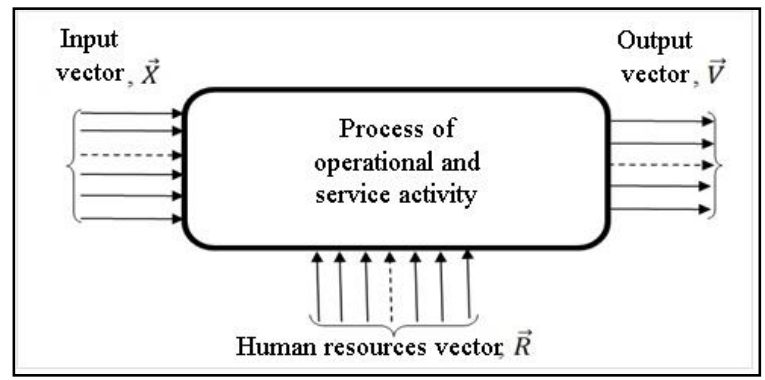

Fig. 2. Representation of process of operational and service activity of fire service's personnel subsystems in terms of "input - human resources - output" [4]

Let's assume that the target function (7) grows monotonically in the range of $0<R_{k}<\infty$, that is, the more human resources is provided to the fire service in the cluster, the greater the target function, and

i.e. the function is limited from above.

$$
\varphi_{k}\left(F_{k}, \infty, \gamma_{k}\right)=A_{k}=\text { const, }
$$

The target function of the general system of fire service in Vietnam $\Phi_{u}$ is determined by the target functions of its units in clusters and is written as the sum of them: 


$$
\Phi_{u}=\sum_{k=1}^{k=K} \varphi_{k}
$$

Having limited human resources the center seeks to achieve optimal value of its system target (general target function) by defining target functions of active elements (fire services in clusters) by choosing such a distribution of human resources that stimulates them to improve ways and methods of operational activities in extinguishing fires, intensifying the use of available human resources.

The task of distribution of human resources with known dependence $\varphi_{k}\left(F_{k}, R_{k}, \bar{\gamma}_{k}\right)$ and known parameters $\gamma_{\mathrm{k}}$ is set as the following optimization target

$$
\Phi_{u}=\sum_{k=1}^{k=K} \varphi_{k}\left(F_{k}, R_{k}, \bar{\gamma}_{k}\right) \rightarrow \max (\bar{R}),
$$

with the restriction in the number of human resources at the Center's disposal:

$$
\sum_{k=1}^{k=K} R_{k}=R
$$

The principle, which is implemented in the formulation of the task of distribution of fire service's human resources in clusters, is the principle of optimal distribution.

Let's consider analytical dependence (6) reflecting the relationship between specific load by fires and complex specific indicator by weighted number of deaths and injuries in fires to form the target function:

$$
s_{k}=0,318 \cdot \exp \left(101,04 \cdot h_{k}\right),
$$

where the $h_{k}$ is the number of fires per firefighter in the $k$-th cluster.

It is evident that the problem of optimal distribution of human resources in clusters should be set so as to minimize the amount of quantities (12) throughout all $k=1, \ldots, K$; or to maximize the amount of their reciprocal values:

$$
1 / s_{k}=3,145 \cdot \exp \left(-101,04 \cdot h_{k}\right) \text {. }
$$

Let's then define the target function:

$$
\varphi_{k}=3,145 \cdot\left[1-\exp \left(-101,04 \cdot h_{k}\right)\right] \approx 3,2 \cdot\left[1-\exp \left(-100 \cdot h_{k}\right)\right] .
$$

which has the following graphic view (Figure 3 ).

It is not too difficult to see that the function (14) increases monotonically, and at $h_{k} \rightarrow \infty, \varphi_{k} \rightarrow 3,2$.

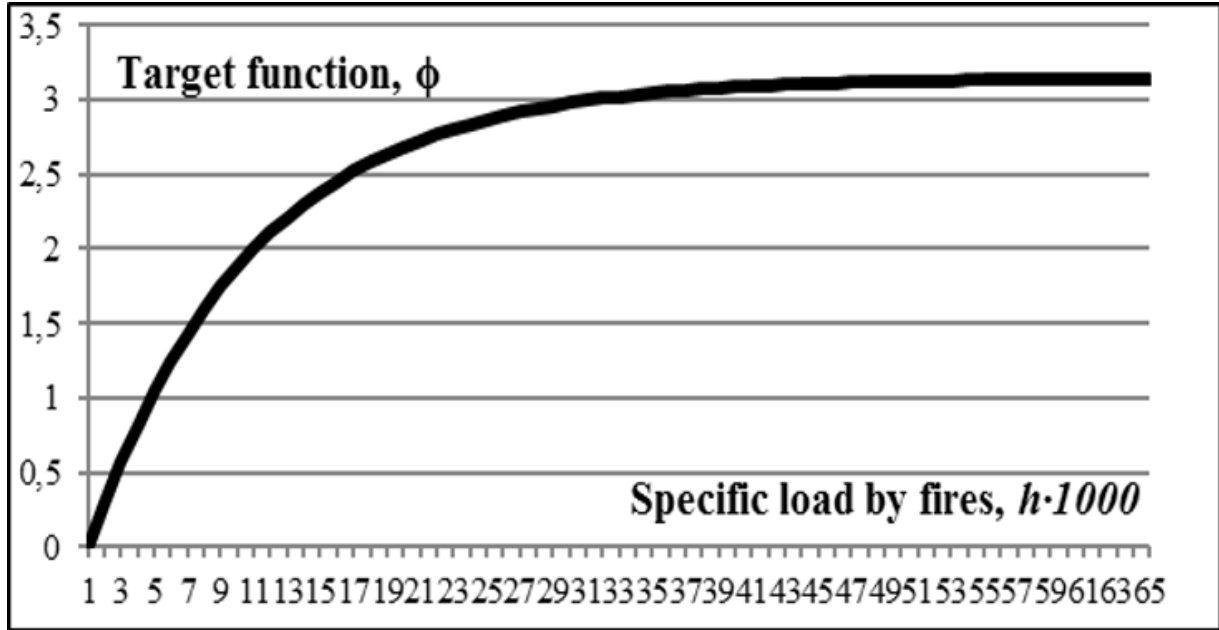

Fig. 3. The target function by complex specific indicator of the number of fatalities and injuries in fires depending on the fire specific load

Expanding (14) in the Maclaurin series, the target function of the Center is represented as follows:

$$
\Phi_{u} \approx \sum_{k=1}^{k=K} 320 \cdot h_{k}\left(1-50 \cdot h_{k}\right) .
$$


The problem of optimal distribution of human resources between the $K$ clusters is set as follows:

$$
\begin{gathered}
\Phi_{u_{R}} \rightarrow \min , \\
\sum_{k=1}^{k=K} R_{k}=R .
\end{gathered}
$$

Let's solve the problem (16) - (17) using Lagrange multipliers method.

The Lagrange function is written as follows:

$$
L\left(\Phi_{u}\right)=\sum_{k=1}^{k=K}\left[320 \cdot h_{k} \cdot\left(1-50 \cdot h_{k}\right)\right]-\lambda_{k} \cdot\left(\sum_{k=1}^{k=K} R_{k}-R\right),
$$

where $\lambda$ - the Lagrange multiplier.

The [6] shows that by differentiating (18) we obtain a cubic equation of optimal solution of which is as follows:

$$
R_{k(o p t)}=R \cdot \frac{\sqrt[3]{F_{k}^{2}}}{\sum_{k=1}^{k=K} \sqrt[3]{F_{k}^{2}}} ; k=1,2, \ldots, K .
$$

Computational experiments showed that using method of optimal distribution of human resources in accordance with (19) would reduce a complex specific indicator by about 10$12 \%$ as compared to the current situation of their distribution in cluster.

\section{Conclusions}

Optimal ensuring of human resources is one of the central tasks, which is related to management of fire service in Vietnam. Finding a solution to this problem is directly related to improving the fire situation in all sectors of the economy, including the construction sector. Representation based on the approaches of theory of active systems [7], process of operational and service activity of fire service's personnel subsystems in clusters in terms of "input - human resources - output" and formal description of "operational and service technology" in fire service's activities turned out to be very constructive for the solution of this problem.

With regard to description of the management model of fire service's human resources in clusters in general, it is necessary to solve the task of multi-vector optimization. A special target function is constructed in this article, which is based on stable dependence of complex specific indicator of the number of deaths and injuries in fires per one firefighter with specific load by fires.

Existing distributions of territorial human resources in clusters in Vietnam (proportional and on the "upon achieved" principle) lose in comparison with the approach of the optimal distribution of human resources in the application of the nonlinear model, which is considered in the article. Computational experiments have shown that using the methodology of optimal distribution of human resources would reduce the specific complex indicator of the number of deaths and injuries in fires by about 10-12\% in the comparison with the current situation of their distribution in clusters in Vietnam.

\section{References}

1. N. Brushlinsky, V. Esin, V. Sluev et al. Fire risks. Issue. 4. Fire risk management. Ed. N. Brushlinsky, N. Shebeko (2006)

2. C. Jennings. Social and economic characteristics as determinants of residential fire risk in urban neighborhoods: A review of the literature. Fire Safety Journal . Part A(0), № 62. Pp.13-19. (2013)

3. N. Benichou., A. Kashef. How to Use Fire Risk Assessment Tools to Evaluate Performance-Based Designs. CIB World Building Congress (2004) 
4. V. Minaev, N. Topolsky, Chu Quoc Minh. Fire risk management based on the theory of active systems. Fires and Emergencies: Prevention, Liquidation, 4 (2014)

5. V. Minaev, N. Topolsky, Dao Anh Tuan. Typologization of the territories of Vietnam on the characteristics of fire danger. Fires and Emergencies: Prevention, Liquidation, 1 (2018)

6. Dao Anh Tuan, V. Minaev, N. Topolsky, A. Faddeev Fire risks. Typology of territories. Resource management (2018)

7. V. Burkov, D. Novikov. How to manage projects: a scientific and practical publication (1997) 